EPJ Web of Conferences 13, 05002 (2011)

DOI: $10.1051 /$ epjconf/20111305002

(c) Owned by the authors, published by EDP Sciences, 2011

\title{
Nonextensive critical effects in relativistic nuclear mean field models ${ }^{\star}$
}

\author{
Jacek Rożynek ${ }^{\mathrm{a}}$ and Grzegorz Wilk ${ }^{\mathrm{b}}$ \\ The Andrzej Sołtan Institute for Nuclear Studies, Hoża 69, 00681 Warsaw, Poland
}

\begin{abstract}
We present a possible extension of the usual relativistic nuclear mean field models widely used to describe nuclear matter towards accounting for the influence of possible intrinsic fluctuations caused by the environment. Rather than individually identifying their particular causes we concentrate on the fact that such effects can be summarily incorporated in the changing of the statistical background used, from the usual (extensive) Boltzman-Gibbs one to the nonextensive taken in the form proposed by Tsallis with a dimensionless nonextensivity parameter $q$ responsible for the above mentioned effects (for $q \rightarrow 1$ one recovers the usual BG case). We illustrate this proposition on the example of the QCD-based Nambu - Jona-Lasinio (NJL) model of a many-body field theory describing the behavior of strongly interacting matter presenting its nonextensive version. We check the sensitivity of the usual NJL model to a departure from the BG scenario expressed by the value of $|q-1|$, in particular in the vicinity of critical points.
\end{abstract}

\section{Introduction}

In all studies of relativistic properties of nuclear matter, mean field models are usually the models of first choice $[1,2]$. These models use a statistical approach based on Boltzmann-Gibbs (BG) statistics which is, strictly speaking, only correct when the corresponding heat bath is homogeneous and infinite. These conditions are by no means met in realistic situations in which nuclear matter occurs. Usually one encounters some inherent problems arising, for example, from the smallness of the collisional systems and their rapid evolution. These, among other things, render the spatial configuration of the system far from uniform and prevent global equilibrium from being established (cf. [3] and references therein). As a result, some quantities become non extensive and develop power-law tailed rather than exponential distributions. The widely used way to account for these effects is to resort to a nonextensive statistics, known as $q$-statistics [4]. The new phenomenological nonextensivity parameter $q$ occurring there is supposed to account for all possible dynamical factors violating the assumptions of the usual BG statistics. This is recovered in the limit of $q \rightarrow 1$. Because it enters into the respective formulas of the particular dynamical model used for a given investigation, it allows for a simple phenomenological check of the stability of the model against possible deviations from the BG approach.

So far, applications of the nonextensive approach are numerous and cover all branches of physics [5]. These include high energy multiparticle production processes (cf., [6]) and different aspects of nuclear and quark matter [79]. The nonextensive framework can also be derived from

\footnotetext{
^ Presented by G.Wilk

a e-mail: rozynek@fuw.edu.pl

b e-mail:wilk@fuw.edu.pl
}

a special treatment of kinetic theory investigating complex systems in their nonequilibrium stationary states [10]. Some examples of more specialized topics can be found in [11] and references therein. For an illustration, the Tsallis distribution, $h_{q}(E)$, and $\mathrm{BG}$ distribution, $f(E)$, are connected as follows:

$$
\begin{aligned}
h_{q}(E) & =\exp _{q}\left(-\frac{E}{T}\right)=\frac{2-q}{T}\left[1-(1-q) \frac{E}{T}\right]^{\frac{1}{1-q}} \\
& \stackrel{q \rightarrow 1}{\Longrightarrow} f(E)=\frac{1}{T} \exp \left(-\frac{E}{T}\right) .
\end{aligned}
$$

It is usually argued that, for the $q>1$ case, $q-1$ is a measure of intrinsic fluctuations of the temperature in the system considered [12], whereas $q<1$ is usually attributed to some specific correlations limiting the available phase space [13] or to the possible fractality of the allowed phase space [14] (other possible interpretations were considered in $[11])^{1}$.

For our further considerations of importance are recent applications of nonextensive statistics in description of nuclear [18] and quarkonic matter [8,19,20], the later of which we shall continue here. In [18], the $q$-version of the Walecka many-body field theory [1] has been investigated. It was shown there that $q$-statistics results in the en-

\footnotetext{
${ }^{1}$ One must admit at this point that this approach is subjected to a rather hot debate of whether it is consistent with the equilibrium thermodynamics or else it is only a handy way to a phenomenological description of some intrinsic fluctuations in the system [16]. It should be therefore noticed that it was demonstrated on general grounds [17] that fluctuation phenomena can be incorporated into a traditional presentation of a thermodynamic. The Tsallis distribution (1) belongs to the class of general admissible distributions which satisfy thermodynamical consistency conditions and which are therefore a natural extension of the usual BG canonical distribution (2).
} 
hancement of the scalar and vector meson fields in nuclear matter, in diminishing of the nucleon effective mass and in hardening of the nuclear equation of state (only the $q>1$ case was considered there). In [8] the relativistic equation of state of hadronic matter and a quark-gluon plasma at finite temperature and baryon density was investigated in the framework of nonextensive statistical mechanics. In our work [19] we investigated a nonextensive version of another mean field theory, namely the QCD-based Nambu - Jona-Lasinio (NJL) model of a many-body field theory describing the behavior of strongly interacting matter presented recently in [21]. This time, unlike in [18], we used the quark rather than the hadronic degrees of freedom and, because of this, we had to consider both the $q>1$ and $q<1$ cases. This $q$-NJL model allowed us to discuss the $q$-dependence of the chiral phase transition in dense quark matter, in particular the quark condensates and the effective quark masses and their influence on the masses of $\pi$ and $\sigma$ mesons and on the spinodal decomposition (cf., [19] for details). These results helped us proceed further and consider critical phenomena in strongly interaction matter using $q$-statistics (these phenomena are of interest nowadays, cf., for example, [23,24], but were so far not investigated in non-equilibrium environment provided by $q$-statistics). In particular, we shall now concentrate on the influence of dynamical factors causing nonextensivity and represented by the parameter $q$ in the vicinity of the critical end point (CEP).

\section{Basic elements of the $q$-NJL model}

First we present the basic elements of the $q$-NJL model introduced in [19] (to which we refer for more details).

\subsection{The usual NJL model}

We start with the usual QCD based NJL model based on BG statistics discussed in [21]. It is the standard $S U(3)$ NJL model with $U(1)_{A}$ symmetry described in, with the usual Lagrangian of the NJL model used in a form suitable for the bosonization procedure (with four quarks interactions only), from which we obtain the gap equations for the constituent quark masses $M_{i}$ :

$$
M_{i}=m_{i}-2 g_{s}\left\langle\bar{q}_{i} q_{i}\right\rangle-2 g_{D}\left\langle\bar{q}_{j} q_{j}\right\rangle\left\langle\bar{q}_{k} q_{k}\right\rangle,
$$

with cyclic permutation of $i, j, k=u, d, s$ and with the quark condensates given by $\left\langle\bar{q}_{i} q_{i}\right\rangle=-i \operatorname{Tr}\left[S_{i}(p)\right]\left(S_{i}(p)\right.$ is the quark Green function); $m_{i}$ denotes the current mass of quark of flavor $i$. We consider a system of volume $V$, temperature $T$ and the $i^{\text {th }}$ quark chemical potential $\mu_{i}$ characterized by the baryonic thermodynamic potential of the grand canonical ensemble (with quark density equal to $\rho_{i}=$ $N_{i} / V$, the baryonic chemical potential $\mu_{B}=\frac{1}{3}\left(\mu_{u}+\mu_{d}+\mu_{s}\right)$ and the baryonic matter density as $\left.\rho_{B}=\frac{1}{3}\left(\rho_{u}+\rho_{d}+\rho_{s}\right)\right)$,

$$
\Omega\left(T, V, \mu_{i}\right)=E-T S-\sum_{i=u, d, s} \mu_{i} N_{i}
$$

The internal energy, $E$, the entropy, $S$, and the particle number, $N_{i}$, are given by [21] (here $\left.E_{i}=\sqrt{M_{i}^{2}+p^{2}}\right)$ :

$$
\begin{aligned}
E= & -\frac{N_{c}}{\pi^{2}} V \sum_{i=u, d, s}\left[\int p^{2} d p \frac{p^{2}+m_{i} M_{i}}{E_{i}}\left(1-n_{i}-\bar{n}_{i}\right)\right]- \\
& -g_{S} V \sum_{i=u, d, s}\left(\left\langle\bar{q}_{i} q_{i}\right\rangle\right)^{2}-2 g_{D} V\langle\bar{u} u\rangle\langle\bar{d} d\rangle\langle\bar{s} s\rangle \\
S= & -\frac{N_{c}}{\pi^{2}} V \sum_{i=u, d, s} \int p^{2} d p \cdot \tilde{S}, \\
& \text { where } \quad \tilde{S}=\left[n_{i} \ln n_{i}+\left(1-n_{i}\right) \ln \left(1-n_{i}\right)\right]+ \\
& +\left[n_{i} \rightarrow 1-\bar{n}_{i}\right] \\
N_{i}= & \frac{N_{c}}{\pi^{2}} V \int p^{2} d p\left(n_{i}-\bar{n}_{i}\right) .
\end{aligned}
$$

The quark and antiquark occupation numbers are, respectively,

$$
\begin{aligned}
n_{i} & =\frac{1}{\left\{\exp \left[\beta\left(E_{i}-\mu_{i}\right)\right]-1\right\}}, \\
\bar{n}_{i} & =\frac{1}{\left\{\exp \left[\left(\beta\left(E_{i}+\mu_{i}\right)\right]+1\right\}\right.},
\end{aligned}
$$

and with them one calculates values of the quark condensates present in Eq. (3),

$$
\left\langle\bar{q}_{i} q_{i}\right\rangle=-\frac{N_{c}}{\pi^{2}} \sum_{i=u, d, s}\left[\int \frac{p^{2} M_{i}}{E_{i}}\left(1-n_{i}-\bar{n}_{i}\right)\right] d p .
$$

Eqs. (3) and (10) form a self consistent set of equations from which one gets the effective quark masses $M_{i}$ and values of the corresponding quark condensates.

The values of the pressure, $P$, and the energy density, $\epsilon$, are defined as:

$$
\begin{gathered}
P\left(\mu_{i}, T\right)=-\frac{\Omega\left(\mu_{i}, T\right)}{V}, \quad \epsilon\left(\mu_{i}, T\right)=\frac{E\left(\mu_{i}, T\right)}{V} \\
\text { with } \quad P(0,0)=\epsilon(0,0)=0 .
\end{gathered}
$$

\subsection{The $q$ extension of the NJL model - the $q$-NJL}

The $q$-statistics is introduced by using the $q$-form of quantum distributions for fermions $(+1)$ and bosons $(-1)$ in Eqs. (8) and (9). This is done following a prescription provided in [15], namely by replacing $n$ and $\bar{n}$ by

$$
n_{q i}=\frac{1}{\tilde{e}_{q}\left(\beta\left(E_{q i}-\mu_{i}\right)\right) \pm 1},
$$

(the important point to notice is that one encounters here $E_{q i}=\sqrt{M_{q i}^{2}+p^{2}}$, i.e., that because of $M_{q i}$ also energy is now a $q$-dependent quantity). Denoting $x=\beta(E-\mu)$ one has that for $q>1$

$$
\tilde{e}_{q}(x)=\left\{\begin{array}{lll}
{[1+(q-1) x]^{\frac{1}{q-1}}} & \text { if } & x>0 \\
{[1+(1-q) x]^{\frac{1}{1-q}}} & \text { if } & x \leq 0
\end{array},\right.
$$


whereas for $q<1$

$$
\tilde{e}_{q}(x)=\left\{\begin{array}{lll}
{[1+(q-1) x]^{\frac{1}{q-1}}} & \text { if } & x \leq 0 \\
{[1+(1-q) x]^{\frac{1}{1-q}}} & \text { if } & x>0
\end{array} .\right.
$$

This is because only then can one consistently treat on the same footing quarks and antiquarks (and for all values of $x$ ). This should show the particle-hole symmetry observed in the $q$-Fermi distribution in plasma containing both particles and antiparticles, namely that

$$
n_{q}(E, \beta, \mu, q)=1-n_{2-q}(-E, \beta,-\mu) .
$$

This means, therefore, that in a system containing both particles and antiparticles (as in our case) both $q$ and $2-q$ occur (or, when expressed by a single $q$ only, one can encounter both $q>1$ and $q<1$ at the same time). These dual possibilities warn us that not only $q>1$ but also $q<1$ (or $(2-q)>1$ have physical meaning in the systems we are considering. This differentiates our $q$-NJL model from the $q$-version of the model presented in [18]. Notice that for $q \rightarrow 1$ one recovers the standard FD distribution, $n(\mu, T)$. Actually, it is important to realize that for $T \rightarrow 0$ one always gets $n_{q}(\mu, T) \rightarrow n(\mu, T)$, irrespectively of the value of $q$ [18]. This means that we can expect any nonextensive signature only for high enough temperatures (how high depends on circumstances and on the kind of observable considered, for illustration of this point see results presented in our paper [19] and Fig. 2 below).

Our $q$-NJL model is then obtained by replacing the formulas of Section 2.1 with their $q$-counterparts in what concerns the form of the FD distributions. Additionally, when calculating energies and condensates we follow $[7,8]$ and use the $q$-versions quark condensates, replacing Eqs. (10), (3) and (5) by their $q$-forms:

$$
\begin{gathered}
\left\langle\bar{q}_{i} q_{i}\right\rangle_{q}=-\frac{N_{c}}{\pi^{2}} \sum_{i=u, d, s}\left[\int \frac{p^{2} M_{q i}}{E_{q i}}\left(1-n_{q i}^{q}-\bar{n}_{q i}^{q}\right)\right] d p \\
M_{q i}=m_{i}-2 g_{S}\left\langle\bar{q}_{i} q_{i}\right\rangle_{q}-2 g_{D}\left\langle\bar{q}_{j} q_{j}\right\rangle_{q}\left\langle\bar{q}_{k} q_{k}\right\rangle_{q} \\
E_{q}=-\frac{N_{c}}{\pi^{2}} V \sum_{i=u, d, s}\left[\int p^{2} d p \frac{p^{2}+m_{i} M_{q i}}{E_{q i}}\left(1-n_{q i}^{q}-\bar{n}_{q i}^{q}\right)\right]- \\
-g_{S} V \sum_{i=u, d, s}\left(\left\langle\bar{q}_{i} q_{i}\right\rangle_{q}\right)^{2}-2 g_{D} V\langle\bar{u} u\rangle_{q}\langle\bar{d} d\rangle_{q}\langle\bar{s} s\rangle_{q} .(18
\end{gathered}
$$

On the other hand, again following [7,8], densities which are given by the the $q$-version of Eq. (7) are calculated with $n_{q}$ 's (not with $n_{q}^{q}$, as in (18) and in (16)). The pressure for given $q$ is calculated using the above $E_{q}$ and the $q$-entropy version of Eq. (6) with (cf. [15])

$$
\begin{aligned}
\tilde{S}_{q}= & {\left[n_{q i}^{q} \ln _{q} n_{q i}+\left(1-n_{q i}\right)^{q} \ln _{q}\left(1-n_{q i}\right)\right]+} \\
& +\left\{n_{q i} \rightarrow 1-\bar{n}_{q i}\right\} .
\end{aligned}
$$

Eq. (16) together with the $q$-version of the gap equation, Eq. (17), are the basic equations from which one deduces all results presented here.

\section{Results}

Before presenting our results concerning nonextensive critical effects we shortly repeat the previous results (cf., [19, 20]). In Fig. 1 we present the typical pressure at critical temperature $T_{c r}$ obtained in a $q$-NJL model as a function of compression $\rho / \rho_{0}$ calculated for different values of the nonextensivity parameter $q$ (see [19] for more details on
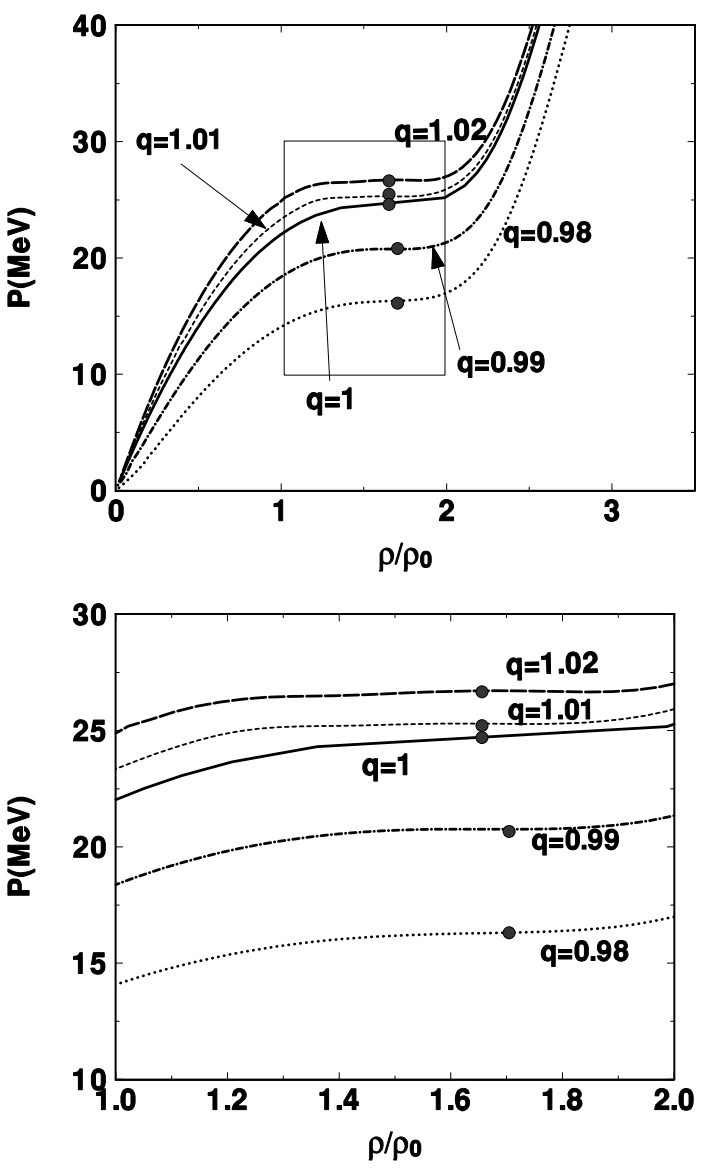

Fig. 1. The pressure at critical temperature $T_{c r}$ as a function of compression $\rho / \rho_{0}$ calculated for different values of the nonextensivity parameter $q$ (the area marked at the upper panel is shown in detail in the lower panel). The dots indicate positions of the inflection points for which first derivative of pressure by compression vanishes. As in [21] for $q=1$ the corresponding compression is $\rho / \rho_{0}=1.67$ (and this leads to $\mu=318.5 \mathrm{MeV}$ ); it remains the same for $q>1$ considered here (but now $\mu=321$ $\mathrm{MeV}$ for $q=1.01$ and $\mu=326.1 \mathrm{MeV}$ for $q=1.02)$ whereas it is shifted to $\rho / \rho_{0}=1.72$ for $q<1(\mu=313 \mathrm{MeV}$ for $q=0.99$ and $\mu=307.7 \mathrm{MeV}$ for $q=0.98$ ).

spinodial decomposition and chiral symmetry restoration in $q$-NJL model) $)^{2}$. Notice that the effect is stronger for

2 There is still an ongoing discussion on the meaning of the temperature in nonextensive systems. However, in our case the small values of the parameter $q$ deduced from the data allow us to argue that, to first approximation, $T_{q}=T$ used here and in [18]. 
$q<1$ and that, essentially, the saddle point remains at the same value of compression. When one moves away from the critical temperature, the typical spinodal structure occurs, which is more pronounced for lower temperatures whereas its sensitivity to the $q$ parameter gets stronger with increasing temperature (cf., [19]). However, it occurs that, for each temperature (even for very small one) a $q>1$ exists for which there is no more mixed phase and for which the spinodal effect vanishes. This seems to be a quite natural effect in the scenario in which $q>1$ is attributed to the fluctuations of the temperature in a system considered as proposed in [12]. On the contrary, effects like correlations or limitations of the phase space considered in [13, 14] work towards an increase of the $T_{c r}$ and make the spinodal effect more pronounced.

A few remarks are in order here (for more detailed discussion we refer to [19]). Nonextensive dynamics enter the NJL calculations through the quark (antiquark) number distribution functions $n_{q i}\left(\bar{n}_{q i}\right)$. These functions are connected with the respective quark (antiquarks) spectral functions in the NJL model. However, deviations from the exponential shape of $q$-exponents, as defined in Eqs. (13) and (14), are negligible for values of $q$ close to unity (in our case $0.98<q<1.02)$. It is also important to notice that Eqs. (13) and (14) are symmetric for $q \leftrightarrow 1-q$. The differences between $q<1$ and $q>1$ cases observed in our results are then due to our way of defining the energy (18) and entropy (19), which, following [7,8], we do by using $n_{q i}^{q}$ and $\bar{n}_{q i}^{q}$ instead of $n_{q i}$ and $\bar{n}_{q i}{ }^{3}$. Because now for $q<1$ distributions $n^{q} q$ and $\bar{n}^{q}{ }_{q i}$ are closer to unity than $n_{q i}$ and $\bar{n}_{q i}$, therefore the absolute values of quark condensates (as given by Eq. (16)) begin to decrease for $q=0.98$ at lower temperature as compared with the $q=1$ case. Therefore the corresponding energy is larger, which means that $q<1$ introduces some residual attractive correlations which rise the energy and lead to hadronization occurring at lower temperature. On the other hand, $q>1$ introduces fluctuations which decrease the effective occupations $\left(n^{q}{ }_{q i}\right.$ and $\bar{n}_{q i}^{q}$ ) and the energy, and smears out the chiral phase transition. In Fig. 2 we present our phase diagram in the $\mu-T$ plane for different nonextensivity parameters considered here with positions of the corresponding critical end points (CEP) for different values of $q$ clearly indicated. The overlap of curves observed in Fig. 2 (inlet) indicates how the critical end point is smeared to a kind of critical area. This is because fireballs created in different events can have different values of $q$ (representing, as mentioned before, action of all factors responsible for the departure of our system from the usual BG approach - not specified here in detail but, in general, resulting in specific correlations of quarks or fluctuations of temperature mentioned

In high energy physics it is just the hadronizing temperature (and instead of the state of equilibrium one deals there with some kind of stationary state). For a thorough discussion of the temperature of nonextensive systems, see [22].

${ }^{3}$ It is worth notice that in [18], which considers only the $q>$ 1 case and uses number distributions without powers of $q$, the significant effects were obtained only for much larger values of the nonextensive parameter $q=1.2$. before). Therefore when analyzing experimental data one most probably will encounter such a critical area instead of a well defined CEP.

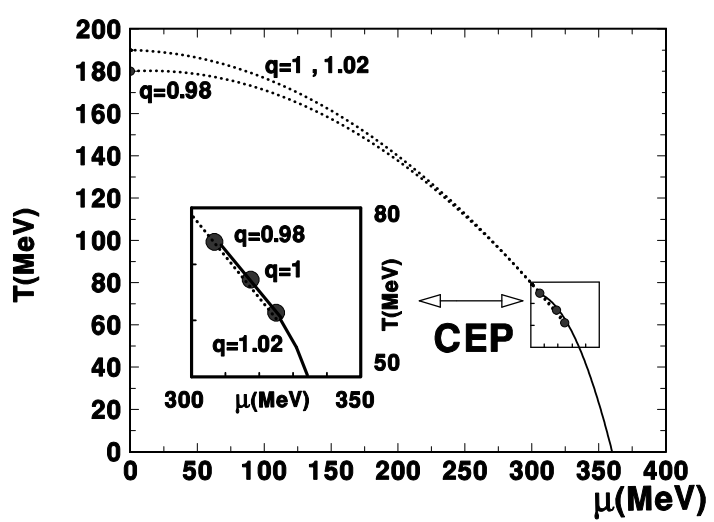

Fig. 2. Phase diagram in the $q$-NJL model in $T-\mu$ plane for values of $q$ considered before: $q=0.98,1.0,1.02$. Solid and dashed lines denote, respectively, first order and crossover phase transitions. The results are presented for three different values of the nonextensivity parameter $q$ with the vicinity of the ( $q$ dependent) critical end points (CEP) enlarged in the inlet. The crossover phase transition for $q=0.98$ and for $\mu \rightarrow 0$ takes place for a smaller temperature $T$.

The role of all these factors is shown in more detail in Fig. 3 which shows the baryon compression $\rho / \rho_{0}$ (calculated in the vicinity of the critical values of temperature and density indicated by the corresponding dotted lines) as the function of the chemical potential $\mu$ for different values of the nonextensivity parameter, $q=0.98,1.00,1.02$. Notice the remarkable difference of the density derivative at the critical point: from the smooth transition through the critical point for $q<1$ to a big jump in density for critical value of chemical potential for $q>1$. It reflects the infinite values of the baryon number susceptibility, $\chi_{B}$ :

$$
\chi_{B}=\sum_{i=u, d, s}\left(\frac{\partial \rho_{i}}{\partial \mu_{B}}\right)_{T}=-\sum_{i=u, d, s}\left(\frac{\partial^{2} \Omega}{\partial^{2} \mu_{B}}\right)_{T} .
$$

The transition between the confined and deconfined phases and/or chiral phase transition [23] can be seen by measuring, event by event, the difference in the magnitude of local fluctuation of the net baryon number in a heavy ion collision [25]. They are initiated and driven mainly by the quark number fluctuation, described here by $\chi_{B}$, and can survive through the freezout [25]. Consequently, our q-NJL model allows us to make the fine tuning for the magnitude of baryon number fluctuations (measured, for example, by the charge fluctuations of protons) and to find the value of the parameter $q$ characteristic for this system. However, it does not allow us to differentiate between possible dynamical mechanisms of baryon fluctuation. We close by noticing that using $q$ dependent $\chi_{B}$ leads to $q$-dependent parameter $\epsilon$ of the critical exponents which describe the 

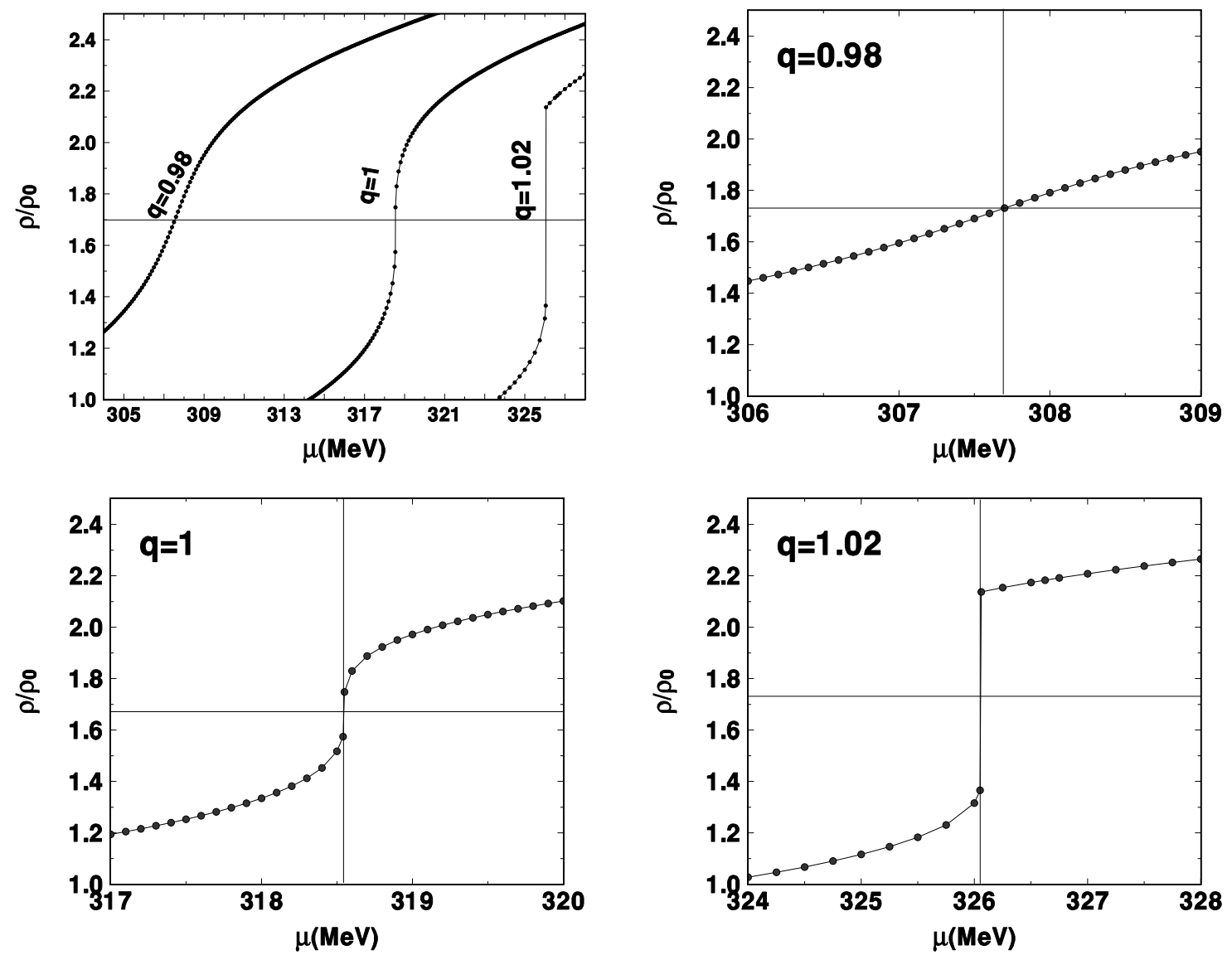

Fig. 3. The baryon compression $\rho / \rho_{0}$ (calculated in the vicinity of the critical values of temperature and density indicated by the corresponding dotted lines) as function of the chemical potential $\mu$ for different values of the nonextensivity parameter, $q=0.98,1.00,1.02$. The summary presented in the top-left panel is detailed in the three consecutive panels.

behavior of baryon number susceptibilities near the critical point [26]. Whereas in the mean field universality class one has $\epsilon=\epsilon^{\prime}=2 / 3$, our preliminary results using the $q$-NJL model show a smaller value of this parameter for $q>1,(\epsilon \sim 0.6$ for $\mathrm{q}=1.02)$ and greater for $q<1(\epsilon \sim 0.8$ for $\mathrm{q}=0.98)$. It would be interesting to deduce the corresponding values of $q$ from different models and compare them with results on a lattice which, by definition, should correspond to $q=1$ (it should be mentioned at this point that there are already attempts to apply Monte Carlo methods, simulating lattice gauge field dynamics as based on non-extensive rather than extensive thermodynamics, see [27] and references therein).

In order to further investigate the $q$ dependence of $\chi_{B}$ let us rewrite Eq. (20) in the following form (recall that $\rho=N_{q} / V$ and $N_{q}$ is $q$-version of Eq. (7)):

$$
\chi_{B}=\frac{1}{\pi^{2}} \sum_{i=u, d, s} \int p^{2} d p\left(\frac{\partial n_{q i}}{\partial \mu_{B}}-\frac{\partial \bar{n}_{q i}}{\partial \mu_{B}}\right)_{T} .
$$

The $q$-versions of occupation numbers, $n_{q i}$ and $\bar{n}_{q i}$, are taken from Eq. (12). The $q$-version of energies there depend on masses $M_{q i}$, which are given by gap equation (17) in a quite involved way. Therefore, the $q$-dependence enters here in two ways: by rather straightforward replacement of $\exp (. .$. by the respective $\exp (. .$.$) in Eq. (12) and by quite involved$ $q$-dependence of $M_{q i}$ given by the gap equation (17). Therefore,

$$
\chi_{B}\left(\mu_{B}, T\right)=\frac{1}{\pi^{2} T} \cdot\left[\chi\left(\mu_{B}\right)+\bar{\chi}\left(\mu_{B}\right)\right]
$$

with

$$
\begin{aligned}
& \chi\left(\mu_{B}\right)=\sum_{i=u, d,}\left[\int p^{2} d p n_{q i}^{2}\left(\frac{1-n_{q i}}{n_{q i}}\right)^{f(q)}\left(1-\frac{M_{q i}}{E_{q i}} \frac{\partial M_{q i}}{\partial \mu_{B}}\right)\right], \\
& \bar{\chi}\left(\mu_{B}\right)=\sum_{i=u, d,}\left[\int p^{2} d p \bar{n}_{q i}^{2}\left(\frac{1-\bar{n}_{q i}}{\bar{n}_{q i}}\right)^{f(q)}\left(1+\frac{M_{q i}}{E_{q i}} \frac{\partial M_{q i}}{\partial \mu_{B}}\right)\right],
\end{aligned}
$$

where

$$
\begin{aligned}
& f(q)=(2-q) \quad \text { if } \quad(q-1)\left(E_{q i}-\mu_{B}\right)>0, \\
& f(q)=q . \quad \text { otherwise. }
\end{aligned}
$$

Our results are presented in Figs. 4 and 5. It turns out that the chiral phase transition investigated here (Fig. 5) is mainly driven by the behavior of the light quark mass 

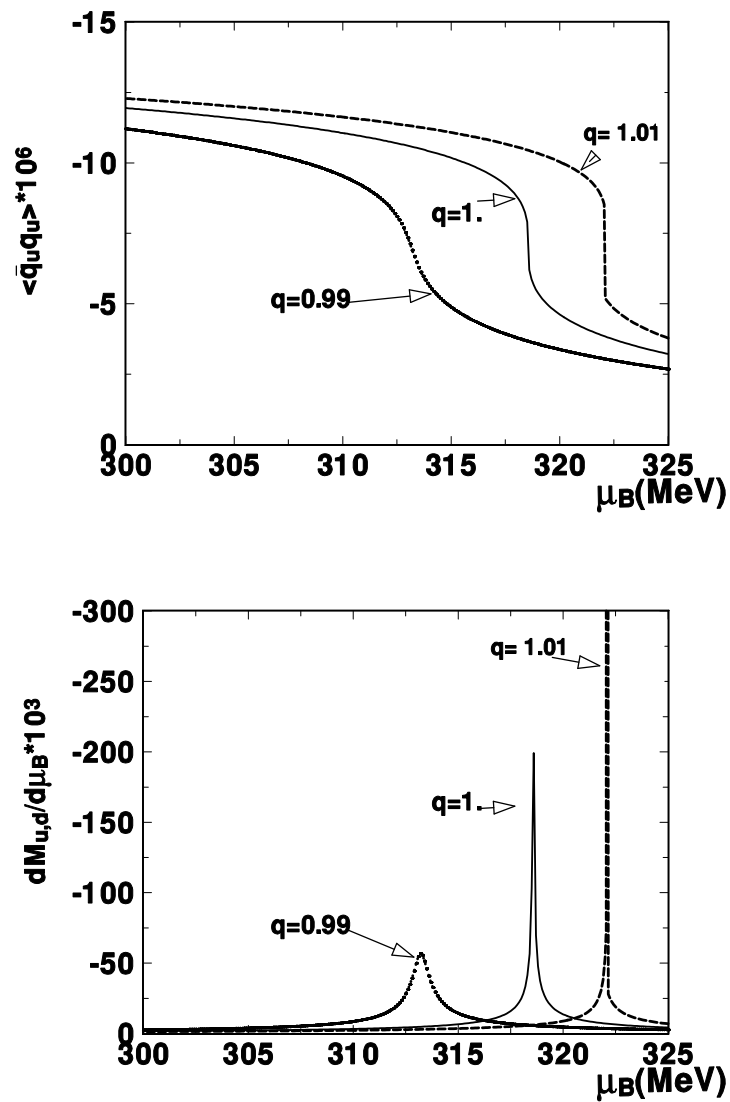

Fig. 4. Upper panel: the chemical potential $\left(\mu_{B}\right)$ dependence of the light quarks condensate in the vicinity of the critical region calculated according to Eq. (16) for different values of the nonextensivity parameter $q: q=0.99,1.0$ and 1.01. Bottom panel: the $\mu_{B}$ dependence of the chemical potential derivative of the light quark mass $M_{q u}$ calculated according to Eq. (17) in the critical region for the same values of $q$ as above.

derivative, see Fig. 4, which in turn is determined by the behavior of the light condensate, cf., Fig. 3. Thus the dynamic of the nonextensive effects is generated not so much by the nonextensive form of occupation numbers in Eq. (12) but rather by the main gap equation (3) where both the condensates and the effective quark masses are present.

\section{Summary}

We have investigated the sensitivity of critical behavior of the QCD based NJL type of mean theory type presented in [21], the $q$-NJL model, to the departure from the conditions required by the application of the $\mathrm{BG}$ approach by using the Tsallis version of nonextensive statistical mechanics [4]. All factors causing this departure are summarily described by the nonextensivity parameter $q$, such that $q-1$ quantifies departure from the BG situation (which is recovered for $q \rightarrow 1$ ).

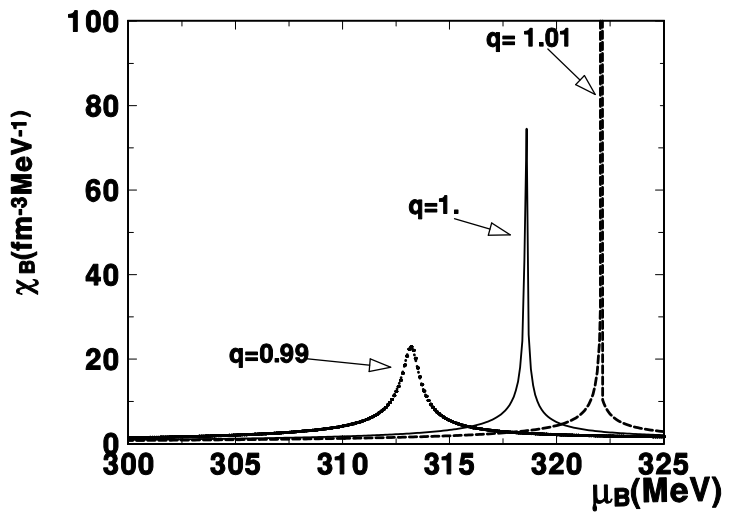

Fig. 5. The $\mu_{B}$ dependence of the baryon number susceptibility, $\chi_{B}$, in the vicinity of the critical region, calculated according to Eq. (21) for different nonextensivity nonextensive parameters, $q=0.99,1.0$ and 1.01. Notice that it is essentially identical with results presented in the bottom panel of Fig. 4 .

We have investigated two possible scenarios corresponding to $q>1$ and $q<1$, respectively, which, as mentioned, correspond to different physical interpretations of the nonextensivity parameter. For $q<1$ (usually connected with some specific correlations [13] or with fractal character of the phase space [14]) we observe a decreasing of pressure, which reaches negative values for a broad $(q$ dependent) range of temperatures and increasing of the critical temperature ${ }^{4}$. The $q>1$ case (usually connected with some specific nonstatistical fluctuations existing in the system [12]) we observe a decreasing of the critical temperature, $T_{c r i t}$, and therefore in the limit of large $q$ we do not have a mixed phase but rather a quark gas in the deconfined phase above the critical line (on the contrary, the compression at critical temperature does not depend on $q$. As in [18] the resulting equation of state is stiffer (in the sense that for a given density we get larger pressure with increasing $q$ ). As expected, the effects depend on the temperature, and tend to vanish when the temperature approaches zero. Fig. 3 shows that the nonequilibrium statistics dilutes the border between the crossover and the first order transition. Finally, Figs. 4 and 5 demonstrate that the most important $q$-dependence is coming from the main gap equation (3), where both the condensates and the effective quark masses are present, rather than from the the nonextensive form of occupation numbers in Eq. (12).

We would like to end by stressing that our results could be of interest for investigations aimed at finding the critical point in high energy heavy ion collisions [3] or when studying particularities of the equation of state $(\mathrm{EoS})$ of compact stars [29]. The fact that they depend on the parameter $q$ means that the exact position of such a point or the type or shape of EoS could be quite different from what is naively expected.

\footnotetext{
${ }^{4}$ It acts therefore in the same way as including of the Polyakov loop into the NJL model [28].
} 


\section{Acknowledgements}

Partial support of the Ministry of Science and Higher Education under contract DPN/N97/CERN/2009 for (GW) and under the Research Project No. N N202046237 for (JR) is acknowledged.

\section{References}

1. J. D. Walecka, Ann. Phys. 83 (1974) 491; S. A. Chin and J. D. Walecka, Phys. Lett. B 52 (1974) 1074; B. D. Serot and J. D. Walecka, Adv. Nucl. Phys. 16 (1986) 1.

2. Y. Nambu and G. Jona-Lasinio, Phys. Rev. 122 (1961) 345 and Phys. Rev. 124 (1961) 246; see also: P. Rehberg, S. P. Klevansky and J. Hüfner, Phys. Rev. C 53 (1996) 410.

3. J. Randrup, Phys. Rev. C 79 (2009) 054911; L. F. Palhares, E. S. Fraga and T. Kodama, J. Phys. G 37 (2010) 094031. See also V. V. Skokov and D. N. Voskresensky, JETP Lett. 90 (2009) 223 and Nucl. Phys. A 828 (2009) 401.

4. C. Tsallis, J. Stat. Phys. 52 (1988) 479; S. R. A. Salinas and C. Tsallis (Eds.), Special Issue on Nonextensive Statistical Mechanics and Thermodynamic, Braz. J. Phys. 29 (1999); M. Gell-Mann and C. Tsallis (Eds.) Nonextensive Entropy Interdisciplinary Applications (Oxford University Press, New York) (2004); C. Tsallis, Eur. Phys. J. A 40 (2009) 257.

5. M. Gell-Mann and C. Tsallis (Eds.), Nonextensive Entropy - interdisciplinary applications (Oxford University Press) (2004); J. P. Boon and C. Tsallis (Eds.), Nonextensive Satistical Mechanics: New Trends, New Perspectives, Europhysics News 36 (2005) (special issue). An updated bibliography on Tsallis nonextensive statistics can be found at http://tsallis.cat.cbpf.br/biblio.htm.

6. G. Wilk and Z. Włodarczyk, Eur. Phys. J. A 40 (2009) 299; T. Osada and G. Wilk, Phys. Rev. C 77 (2008) 044903 and Centr. Eur. J. Phys. 7 (2009) 432; M. Biyajima, T. Mizoguchi, N. Nakajima, N. Suzuki and G. Wilk, Eur. Phys. J. C 48 (2006) 597.

7. W. M. Alberico and A. Lavagno, Eur. Phys. J. A 40 (2009) 313; A. Drago, A. Lavagno and P. Quarati, Physica A 344 (2004) 472.

8. A. Lavagno, D. Pigato and P. Quarati, J. Phys. G 37 (2010) 115102.

9. T. S. Biró, K. Ürmösy and Z. Schram, J. Phys. G 37 (2010) 094027.

10. T. S. Biró and G. Purcsel, Centr. Eur. J. Phys. 7 (2009) 395; T. S. Biró, G. Purcsel and K. Ürmos̈y, Eur. Phys. J. A 40 (2009) 325; T. S. Biró and G. Purcsel, Phys. Rev. Lett. 95 (2005) 162302 and Phys. Lett. A 372, (2008) 1174; T. S. Biró, Europhys. Lett. 84 (2008) 56003; G. Kaniadakis, Eur. Phys. J. A 40 (2009) 275 and Eur. Phys. J. B 70 (2009) 3.

11. H. Hasegawa, Physica A 388 (2009) 2781 and Phys. Rev. E 80 (2009) 011126; M. S. Reis, V. S. Amaral, R. S. Sarthour and I. S. Oliveira, Phys. Rev. B 73
(2006) 092401; P. Douglas, S. Bergamini and F. Renzoni F, Phys. Rev. Lett. 96 (2006) 110601; R. Silva, G. S. França, C. Vilar and J. S. Alcaniz, Phys. Rev. E 73 (2006) 026102; Du Jiulin, Europhys. Lett. 67 (2004) 893; R. Silva and J. S. Alcaniz, Physica A 341 (2004) 208 and Phys. Lett. A 313 (2003) 393.

12. G. Wilk and Z. Włodarczyk, Phys. Rev. Lett. 84 (2000) 1770 and Chaos, Solitons and Fractals 13/3 (2001) 581; T. S. Biró and A. Jakovác, Phys. Rev. Lett. 94 (2005) 132302.

13. T. Kodama, H-T. Elze, C. E. Aguiar and T. Koide, Europhys. Lett. 70 (2005) 439; T. Kodama and T. Koide, Eur. Phys. J. A 40 (2009) 289.

14. V. García-Morales and J. Pellicer, Physica A 361 (2006) 161.

15. A. M. Teweldeberhan, A. R. Plastino and H. C. Miller, Phys. Lett. A 343 (2005) 71; A. M. Teweldeberhan, H. G. Miller and R. Tegen, Int. J. Mod. Phys.\} E 12 (2003) 395. See also, F. Büÿukkiliç and D. Demirhan, Phys. Lett. A 181 (1993) 24; F. Büyükkiliç, D. Demirhan and A. Güleç, Phys. Lett. A 197 (1993) 209.

16. M. Nauenberg, Phys. Rev. E 67 (2003) 036114 and Phys. Rev. E 69 (2004) 038102; C. Tsallis, Phys. Rev. E 69 (2004) 038101; R. Balian and M. Nauenberg, Europhysics News 37 2006) 9; R. Luzzi, A. R. Vasconcellos and J. Galvao Ramos, Europhysics News 37 (2006) 11.

17. O. J. E. Maroney, Phys. Rev E 80 (2009) 061141.

18. F. I. M. Pereira, B. Silva and J. S. Alcaniz, Phys. Rev. C 76 (2007) 015201.

19. J. Rożynek and G. Wilk, J. Phys. G 36 (2009) 125108.

20. J. Rożynek and G. Wilk, Acta Phys. Polon. B 41 (2010) 351.

21. P. Costa, M. C. Ruivo and A. de Sousa, Phys. Rev. D 77, (2008) 096001.

22. S. Abe, Physica A 368 (2006) 430.

23. T. Hatsuda and T. Kunihiro, Phys. Rep. 247 (1944) 221; V. Bernard, U-G. Meissner and I. Zahed, Phys. Rev. D 36 (1987) 819; M. Buballa, Phys. Rep. 407 (2005) 205

24. C. Sasaki, B. Friman and K. Redlich, Phys. Rev. Lett. 99 (2007) 232301 and Phys. Rev. D 75 (2007) 054026; B.-J. Schaefer and J. Wambach, Phys. Rev. D 75 (2007) 085015; B.-J. Schaefer and M. Wagner, Phys. Rev. D 79 (2009) 014018; E. Nakano, B.-J. Schaefer, B. Stokic, B. Friman and K. Redlich, Phys. Lett. B 682 (2010) 401 and references therein.

25. Y. Hatta, M. A. Stephanov, Phys. Rev. Lett. 91 (2003) 102003; S. Gavin arXiv:nucl-th/9908070v1.

26. Y. Hatta and T. Ikeda, Phys. Rev. D67 (2006) 014028.

27. T. S. Biró, K. Urmössy and Z. Schram, J. Phys. G 37 (2010) 094027.

28. P. Costa, C. A. de Sousa, M. R. Ruivo and H. Hansen, Europhys. Lett. 86 (2009) 31001.

29. T. Klähn, D. Blaschke, S. Typel, E. N. E. van Dalen, A. Faessler, C. Fuchs, T. Gaitanos, H. Grigorian, A. Ho, E. E. Kolomeitsev, M. . Miller, G. Röpke G, J. Trümper, D. N. Voskresensky, F. Weber and H. H. Wolter, Phys. Rev. C 74 (2006) 035802. 